\title{
Calcium Phosphate Nanoparticles with an Asymmetric Lipid Bilayer Coating for siRNA Delivery to the Tumor
}

\author{
Jun Li, Yang Yang ${ }^{*}$, and Leaf Huang ${ }^{*}$ \\ Division of Molecular Pharmaceutics, Eshelman School of Pharmacy, University of North Carolina \\ at Chapel Hill, Chapel Hill, NC 27599, USA \\ *Visiting scholar from State Key Laboratory of Biotherapy, West China Hospital, Sichuan \\ University, The People's Republic of China
}

\begin{abstract}
Calcium phosphate $(\mathrm{CaP})$ nanoparticles(NP) with an asymmetric lipid bilayer coating have been designed for targeted delivery of siRNA to the tumor. An anionic lipid, dioleoylphosphatydic acid (DOPA), was employed as the inner leaflet lipid to coat the nano-size $\mathrm{CaP}$ cores, which entrap the siRNA, such that the coated cores were soluble in organic solvent. A suitable neutral or cationic lipid was used as the outer leaflet lipid to form an asymmetric lipid bilayer structure verified by the measurement of NP zeta potential. The resulting NP was named LCP-II with a size of about 25 to $30 \mathrm{~nm}$ in diameter and contained a hollow core as revealed by TEM imaging. PEGylation of NP was done by including a PEG-phospholipid conjugate, with or without a targeting ligand anisamide, in the outer leaflet lipid mixture. The sub-cellular distribution studied in the sigma receptor positive human $\mathrm{H} 460$ lung cancer cells indicated that LCP-II could release more cargo to the cytoplasm than our previous lipid/protamine/DNA (LPD) formulation, leading to a significant ( 40 fold in vitro and $\sim 4$ fold in vivo) improvement in siRNA delivery. Bio-distribution study showed that LCP-II required more PEGylation for MPS evasion than the previous LPD, probably due to increased surface curvature in LCP-II.
\end{abstract}

\section{Keywords}

Asymmetric lipid bilayer; Calcium phosphate; Nanoparticle; siRNA delivery

\section{Introduction}

RNA interference (RNAi) therapeutics, such as siRNA, requires a suitable vehicle for in vivo delivery [1]. An ideal vehicle for cancer therapy should meet at least four major criteria. They include evasion of the mononuclear phagocytic system(MPS), extravasation from the blood circulation into the tumor, diffusion through the extracellular matrix to bind with tumor cells, and escape from the endosome to release the cargo siRNA into the cytoplasm[2]. Therefore, a well protected nanoparticle (NP) modified with a suitable targeting ligand is considered a typical siRNA delivery vehicle to the tumor if the NP diameter is less than $200 \mathrm{~nm}[3,4]$. Two major types of lipid-based NPs have been developed

\footnotetext{
(C) 2011 Elsevier B.V. All rights reserved.

** Corresponding author. leafh@unc.edu; Phone: (919) 843-0736; Fax: (919) 966-0197.
}

Publisher's Disclaimer: This is a PDF file of an unedited manuscript that has been accepted for publication. As a service to our customers we are providing this early version of the manuscript. The manuscript will undergo copyediting, typesetting, and review of the resulting proof before it is published in its final citable form. Please note that during the production process errors may be discovered which could affect the content, and all legal disclaimers that apply to the journal pertain. 
for targeted siRNA delivery, such as lipid nanoparticle (LNP)[5-8] and lipid/polycation/ DNA complex (LPD)[9-11]. Polymers such as transferrin-cyclodextrin polycationare are also effective [12-14].

To improve the cargo release activity of our previous LPD formulation, we have prepared a $\mathrm{pH}$-sensitive calcium phosphate $(\mathrm{CaP})$ core to replace the protamine/DNA core in the LPD formulation [15]. The Lipid/Calcium/Phosphate type I (LCP-I) formulation was protected by PEG tethered with an anisamide ligand for binding to the sigma receptor over-expressing tumor cells. LCP-I showed a 4-fold improvement of the silencing effect in vitro compared to the previous LPD formulation. There was also a significant target gene silencing activity in a xenograft model with no significant elevation of inflammatory cytokines, i.e., IL-6 and IL-12, in the blood. However, the CaP core in LCP-I is highly hydrophilic and requires an un-scalable column method for purification. In addition, many factors on the surface of the nanoparticle can influence blood residence time and organ-specific accumulation [16].Therefore, it is desirable that a variety of lipid can be used as the outer leaflet lipid. Such flexibility in the choice of the lipid can be important for controlling the pharmacokinetics and tissue distribution properties of the NP.

In the current work, we prepared a novel siRNA delivery vehicle by employing an anionic phospholipid, dioleoylphosphatidic acid (DOPA), as a pre-coating reagent during the formation of the nano-sized $\mathrm{CaP}$ core in which siRNA was encapsulated. The lipid coating prevented the $\mathrm{CaP}$ core from aggregation during the centrifugal separation step and rendered it soluble in $\mathrm{CHCl}_{3}$. The resulting NP core was very small $(25-30 \mathrm{~nm})$ and contained a hollow structure. The DOPA layer on the surface of the $\mathrm{CaP}$ core also served as the inner leaflet lipid for the surface lipid bilayer of LCP. Lipids for the outer leaflet could simply be added into the $\mathrm{CaP}$ core solution in $\mathrm{CHCl}_{3}$. Since a PEG-lipid conjugate, with or without a tethered ligand, could be included in the outer leaflet lipids, it was not necessary to perform PEGylation of NP by post-insertion [17]. The improved formulation is named Lipid/ Calcium/Phosphate type II (LCP-II). Significantly different from the previous LCP-I formulation, LCP-II contains an asymmetric lipid structure verified by the measurement of zeta potential. We report here the preparation, properties, and in vitro and in vivo activity of this formulation.

\section{Materials and Methods}

\subsection{The preparation of LCP-II NP}

The information about the materials is shown in the supplementary information. Figure 1 shows a flow diagram for the preparation of siRNA-entrapped LCP-II NPs. The anionic lipid coating $\mathrm{CaP}$ cores were prepared by a water-in-oil micro-emulsion method. Briefly, $300 \mu \mathrm{L}$ of $500 \mathrm{mM} \mathrm{CaCl} 2$ with $100 \mu \mathrm{L}$ of $2 \mathrm{mg} / \mathrm{mL}$ siRNA was dispersed in $15 \mathrm{~mL}$ Cyclohexane/Igepal CO-520 (71/29 V/V) solution to form a very well dispersed water-in-oil reverse micro-emulsion. The phosphate part was prepared by $300 \mu \mathrm{L}$ of $25 \mathrm{mM} \mathrm{Na}_{2} \mathrm{HPO}_{4}$ $(\mathrm{pH}=9.0)$ in a separate $15 \mathrm{~mL}$ oil phase. Two hundred $\mu \mathrm{L}(20 \mathrm{mg} / \mathrm{mL})$ dioleoylphosphatydicacid (DOPA) in chloroform was added to the phosphate phase. After mixing the above two solutions for $20 \mathrm{~min}, 30 \mathrm{~mL}$ of absolute ethanol was added to the micro-emulsion and the mixture was centrifuged at $12,000 \mathrm{~g}$ for at least $15 \mathrm{~min}$ to remove cyclohexane and surfactant. After being extensively washed by ethanol 2-3 times, the pellets were dissolved in $1 \mathrm{~mL}$ chloroform and stored in a glass vial for further modification.

For the preparation of LCP-II NPs, $500 \mu \mathrm{L}$ of CaP core was mixed with $50 \mu \mathrm{L}$ of $10 \mathrm{mM}$ DOTAP/Cholesterol (1:1) or DOPC/Cholesterol (1:1), and50 $\mu \mathrm{L}$ of $3 \mathrm{mM}$ DSPE-PEG-2000 or DSPE-PEG-AA. After evaporating the chloroform, the residual lipid was dispersed in 400 $\mu \mathrm{L}$ of $5 \mathrm{mM}$ Tris- $\mathrm{HCl}$ buffer $(\mathrm{pH}=7.4)$ to form LCP-II NPs. The zeta potential and particle 
size of LCP-II NPs was determined in $1 \mathrm{mM} \mathrm{KCl}$ by a Malvern ZetaSizer Nano series (Westborough, MA). All liposomes were prepared by the thin film hydration method and extruded through a $100 \mathrm{~nm}$ polycarbonate membrane. (In the Results and Discussion section, when either DOTAP or DOPC was mentioned as the outer leaflet lipid, it meant that DOTAP or DOPC was mixed with the same molar amount of Cholesterol. The preparation of LCP-I was described previously [15]. Liposome-protamine-DNA (LPD) NP was prepared as previously described [11]. The details of quantitative detection of trapping efficiency of siRNA in LCP-II NPs and TEM experiments for the NPs can be found in the supplementary information.

\subsection{Measurement of quenching efficiency of a fluorescence labeled lipid in LCP-II}

Rhod-PE was incorporated in the traditional liposomes or in the outer leaflet of LCP-II coating lipid bilayer. In the case of traditional liposomes, DOPC/Cholesterol/Rhod-PE in the molar ratio of 1:1:0.01were prepared by the thin film rehydration method and adjusted in 5 $\mathrm{mM}$ Tris buffer ( $\mathrm{pH} 7.4$ ) to $20 \mathrm{mM}$ of the total lipids. In the case of LCP-II, the preparation of the core was according to the same protocol described in section 2.2, except that the outer leaflet lipid was DOPC/Cholesterol/Rhod-PE(1:1:0.01) instead of DOPC/Cholesterol(1:1). The original fluorescence intensity of the sample was measured by using a fluorometer (Perkin Elmer, USA) after diluting 20 times. Subsequently, $2 \mu \mathrm{L}$ of $0.1 \%$ trypan blue was added and the fluorescence intensity was measured again. Finally, $20 \mu \mathrm{L}$ of $0.1 \%$ Triton $\mathrm{X}-100$ in PBS was added to the above solution and the fluorescence was recorded again. Triplet samples were used for each group.

\subsection{Cellular studies of LCP-II NP}

H460 cells $\left(1 \times 10^{5}\right.$ per well) were seeded in 12-well plates (Corning Inc., Corning, NY) with cover glass for $12 \mathrm{~h}$ before experiment. Cells were treated with different formulations at a concentration of $100 \mathrm{nM}$ for FAM-labeled dsDNA (mimic of siRNA) in serum containing medium at $37{ }^{\circ} \mathrm{C}$ for $3 \mathrm{~h}$. After washed twice with PBS, cells were fixed with $3.8 \%$ paraformaldehyde in PBS at room temperature for $10 \mathrm{~min}$, counterstained with DAPI (Vector Lab, Burlingame, CA), and imaged by using a Leica SP2 confocal microscope. The details of in vitro luciferase gene silencing study are shown in the supplementary information.

\subsection{Bio-distribution study and in vivo gene silencing study of the LCP-II NP}

Female athymic nude mice of age 6-8 weeks were purchased from Charles River Laboratories (Wilmington, MA). All work performed on animals were in accordance with and approved by the University of North Carolina Institutional Animal Care and Use Committee. H460 cells $\left(2 \times 10^{5}\right)$ with luciferase expression were subcutaneously injected into the lower back of female nude mice (about $20 \mathrm{~g}$ ). When the tumor size reached around 600 $\mathrm{mm}^{3}$, mice were intravenously injected with Cy5.5 labeled siRNA in different formulations at a dose of $0.6 \mathrm{mg} / \mathrm{kg}$. Four h later, mice were sacrificed and tissues were collected following by imaging under the IVIS Imaging System (Xenogen Imaging Technologies, Alameda, CA). The image was quantitatively analyzed by the use of the Image $\mathbf{J}$ software. Only the organs of interest were included in the analysis. The details of in vivo luciferase gene silencing study are shown in the supplementary information.

\section{Results and Discussion}

\subsection{The characterizations of LCP-II}

In the preparation of LCP-II, an amphiphilic phospholipid DOPA (see Figure 1) was added into the phosphate part of the reverse micro-emulsion. DOPA is known to strongly interact with Caions at the interface[18]. It is expected that the $\mathrm{CaP}$ core should be coated with 
DOPA because excess Ca should be available on the core surface. The C18:1 chains of DOPA were sufficiently hydrophobic such that the coated cores were soluble in a nonpoplar solvent, i.e., $\mathrm{CHCl}_{3}$, but not in a polar solvent, i.e., EtOH. The insolubility of the coated core in EtOH allowed convenient washing of the cores in EtOH in which excess surfactants, including free DOPA, was soluble and washed away. The final LCP-II formulation was examined by TEM (Figure 2). Both images of NP were obtained without (panel A, C) and with (panel D) negative staining. Since the lipid bilayer is electron transparent, only the CaP cores of the NPs were imaged without the negative stain. In panel $\mathrm{A}$, the image of $\mathrm{CaP}$ cores without staining showed particles with a high electron intensity in the outer part but lower intensity in the inner part, suggesting a hollow structure for the $\mathrm{CaP}$ cores. The size of the cores was about 20-25 nm and the inner hole was about $8-12 \mathrm{~nm}$. This is in contrast with the much larger particle size $(\sim 120 \mathrm{~nm})$ for our previous LCP-I formulation [15]. A table is shown in the supplementary information for comparison. It is speculated that the $\mathrm{CaP}$ precipitation was initiated at the interface of the micro-emulsion to entrap siRNA. Since the volume of the precipitate is smaller than the volume of water in the micro-emulsion, a hollow structure would be formed in the $\mathrm{CaP}$ cores. A cartoon diagram is also shown in Figure 2B to indicate the process of $\mathrm{CaP}$ precipitate formation. Since DOPA is not soluble in aqueous solution, it is highly unlikely that DOPA will pack in the center aqueous core of the hollow $\mathrm{CaP}$ core and the detail discussion can be found in Supplementary Information. The most reasonable location of DOPA is at the surface of the $\mathrm{CaP}$ core which was probably derived from the oil/water interface of the emulsion.

Since the CaP cores were formed in aqueous droplets, the hollow structure of the core probably would provide opportunity to entrap, at least partially, water soluble drugs for targeted delivery. The trapping efficiency of siRNA in LCP-II was approximately $40 \%$. Figure 2D shows the TEM image of LCP-II with uranyl acetate staining. Although the hollowness of the core was not observed, the coating lipid membrane of the NP could be seen by negative staining (arrows in Figure 2D). The overall size of LCP-II was about 25-30 $\mathrm{nm}$ in diameter, which was a little smaller than the hydrodynamic diameter $(40-45 \mathrm{~nm})$ obtained by dynamic light scattering since TEM images were obtained under a dehydrated condition.

The hydrophobic core coated with DOPA provided a variety of choices for the outer leaflet lipid in the bilayer surface to make a water soluble NP for intravenous administration of siRNA. Both neutral lipid, e.g. DOPC, and cationic lipid, e.g. DOTAP, have successfully served as the outer leaflet lipid together with cholesterol. Whittenton et al has prepared asymmetric liposomes which have different lipid leaflet compositions [19]. The inner leaflet was a cationic lipid to encapsulate negatively charged polynucleotides, and neutral lipid was placed on the outer leaflet to decrease non-specific cellular uptake/toxicity. Similar to this structure, an asymmetric lipid bilayer coating structure was hypothesized as shown in Figure 1. The core is different between asymmetric liposomes and the proposed nanoparticle. For an asymmetric liposome, the inside part is aqueous solution. For the proposed nanoparticle in this paper, the inner leaflet lipid is coated on solid calcium phosphate precipitate.

When CaP core mixed with the additional lipids such as DOTAP, DOPC, Chol and DSPEPEG in chloroform, it would not interact with the lipids due to the presence of organic solvent. The formation of asymmetric bilayer occurred when organic solvent was removed from the mixture and exposed to an aqueous solution. A similar self-assemble process also had been used in other phospholipid coating nanoparticles for in vivo imaging [20, 21].

The asymmetric structure was verified by the measurement of zeta potential (Figure $3 \mathrm{~A}$ ) and the quenching efficiency of a fluorescence lipid marker (figure 3B). When the $\mathrm{CaP}$ cores were formulated with DOPC as the outer leaflet lipid, the zeta potential was $-11 \mathrm{mV}$, which 
was close to that $(5 \mathrm{mV})$ of pure DOPC liposomes. However, when DOTAP was employed as the outer leaflet lipid, the surface potential became $+55 \mathrm{mV}$, which was close to that (75 $\mathrm{mV}$ ) of pure DOTAP liposomes. A control formulation was prepared to coat the CaP core with DOPA, the same lipid as the inner one. The resulting NPs became highly negatively charged $(-80 \mathrm{mV})$ which was similar to that $(-98 \mathrm{mV})$ of pure DOPA liposomes. These results indicated that the surface of the NPs was determined solely by the outer leaflet lipid; the inner leaflet lipid DOPA had minimal contribution.

Another experiment was also performed by use of a fluorescence lipid, i.e., rhodamine-PE (Rhod-PE), incorporated into LCP-II or the traditional liposomes. For LCP-II, the labeled lipid was added to the outer leaflet lipid mix such that it served as a label for only the outer leaflet of the bilayer, if LCP-II was indeed coated with an asymmetric lipid bilayer membrane. On the contrary, the fluorescence lipid was randomly distributed between both the outer and inner leaflets in the case of the traditional liposomes. The distribution of the fluorescence label in these two different nanoparticle formulations was measured by quenching with an impermeable dye, trypan blue. The data in Figure 3 B showed that trypan blue quenched $50 \%$ of the fluorescence intensity of the Rhod-PE incorporated in liposomes. However, $90 \%$ of the fluorescence was quenched when Rhod-PE was used to label the outer leaflet lipid in LCP-II formulation. This is consistent with the predicted asymmetric structure of the coating lipid membrane of LCP-II. When the structure of both the liposome and LCP-II was destroyed by the addition of Triton X-100, all of the Rhod-PE would be accessible and quenchable by trypan blue. Indeed, nearly all fluorescence of Rhod-PE was quenched in both nanoparticle formulations. The result of this experiment again supported the asymmetrical structure of the coating lipid bilayer membrane of LCP-II.

It is well known that a PEG layer is necessary to coat the NP to prolong the circulation time and enhance the tumor uptake via the enhanced permeability and retention (EPR) effect[3, 22, 23]. PEG-lipid conjugates, such as DSPE-PEG and DSPE-PEG-AA, could be readily added together with the outer leaflet lipid to form LCP-II. No post-insertion protocol for PEGylation is necessary as commonly done for other lipidic NP formulations [17]. PEG layer on the surface of the NP effectively shields the charges of the outer leaflet lipid. After coating with DOTAP and DSPE-PEG as the outer leaflet lipids, the NPs appeared to be $42-50 \mathrm{~nm}$ (hydrodynamic diameter) in size with a zeta potential of $+5 \mathrm{mV}$. This is to be compared with the NPs without DSPE-PEG in which the zeta potential was $55 \mathrm{mV}$. Thus, PEGylation performed with our method also effectively shielded the surface charge of NP. For targeted delivery of siRNA, DSPE-PEG was replaced by DSPE-PEG-AA. The surface of the NP was covered by a modified anisamide ligand, which contained a secondary amine[24]. Thus, the presence of the target ligand elevated the zeta potential of LCP-II to approximately $+25 \mathrm{mV}$.

We have used HPLC to monitor the stability of the LCP-II formulation in terms of the cleavage of siRNA by serum. The results showed that there was about $5 \%$ of degradation of siRNA with $1 \mathrm{~h}$ incubation. Less than $20 \%$ of siRNA degradation was observed even after 5 h.

\subsection{Sub-cellular distribution of FAM-dsDNA entrapped in LCP-II and gene silencing activity of siRNA delivered by LCP-II}

To achieve ligand meditated endocytosis of siRNA to cells, anisamide, a compound specifically binding to the sigma receptor, was tethered to the distal end of DSPE-PEG as a targeting ligand. FAM-labeled dsDNA as a model for siRNA was entrapped in LCP-II and incubated with $\mathrm{H} 460$ cells for $3 \mathrm{~h}$ to study the subcellular distribution of the delivered siRNA. As shown in Figures 4A and 4B, short arrows indicate that FAM-labeled dsDNA was evenly spread throughout the cytoplasm of $\mathrm{H} 460$ cells after anisamide targeting, but not 
when the LCP-II was not tethered with anisamide. On the contrary, long arrows in Figure 4 $\mathrm{C}$ showed the punctate distribution of FAM-labeled dsDNA delivered by the targeted LPD. This difference was probably because the affinity of protamine and nucleic acid in LPD was too strong to release the cargo to the cytoplasm. The data is consistent with the notion that LCP-II de-assembles in the acidic endosome and releases its cargo into the cytoplasm. The cytoplasmic release activity of LCP-I, a formulation similar to LCP-II, was demonstrated by detecting the increased $\mathrm{Ca}$ ion in the cytoplasm by using a Ca-sensitive dye [15].

H460 cells stably expressing the firefly luciferase were used to examine the siRNA delivery activity of NP. Luciferase siRNA was encapsulated inLPD, LCP-I and LCP-II with either DOPC or DOTAP as the outer leaflet lipid. All of the NPs were modified with DSPE-PEGAA to improve the ligand-meditated endocytosis and the silencing effect on the luciferase activity was detected after $24 \mathrm{~h}$ of the treatment. The dose response curve in Figure 5 showed that the $\mathrm{IC}_{50}$ was 50 and $200 \mathrm{nM}$ in siRNA for LCP-I and LPD, respectively. The $\mathrm{IC}_{50}$ was $5 \mathrm{nM}$ for LCP-II coated with either DOPC or DOTAP, but control siRNA did not show a significant silencing effect. The data indicate that the siRNA could effectively suppress luciferase expression and the potency in siRNA delivery by LCP-II was improved 10-fold as compared to LCP-I, and 40-fold to LPD. From the results, the data also show that the silencing effect was dependent on the CaP core instead of the outer leaflet lipid. Similar to LCP-I, the CaP core in LCP-II also dissolves in low $\mathrm{pH}$ in the endosome to increase the osmotic pressure. The swollen endosome finally bursts and releases the entrapped siRNA, calcium and phosphate into the cytoplasm. Therefore, it does not need cationic lipid to bind with the negatively charged endosome membrane lipid to release the cargo, causing the down-regulation efficiency to become outer leaflet lipid independent. In comparison, in vitro silencing effect of anti-luciferase siRNA formulated inLipofactamine2000®was done. The $\mathrm{IC}_{50}$ was around $20 \mathrm{nM}$ in siRNA concentration. Higher concentrations of control siRNA were formulated by LPD and LCP-I in previous publications $[9,15]$, none of them had any significant down-regulation effect.

\subsection{Tissue Distribution and Tumor Uptake of siRNA}

The bio-distribution of siRNA formulated in LCP-II was studied in a xenograft model of H460 human lung cancer by using Cy5.5-labeled siRNA. We first investigated the effect of PEG density of LCP-II on bio-distribution. It is well established that a PEG brush on the surface of NP effectively resists the uptake of NP by RES [3]. Ordinarily, only 5-6\% PEGylated lipid conjugate can be inserted into the surface of liposomes; higher amounts lead to solubilization of the liposome bilayer and loss of the entrapped cargo [25]. The stealth liposomes contain about 5.6\% surface PEG which is not enough for the formation of a polymer brush [25]. The membrane/core type of NP could carry higher density of PEG on the surface due to the presence of the supported bilayer [26]. For our previous LPD formulation, $10.6 \%$ of DSPE-PEG in the total surface lipid was required to avoid the RES uptake, but still keep the bilayer structure intact because of the existence of a supported bilayer [3]. However, in the LCP-II formulation, liver uptake was still significant even 16\% of the DSPE-PEG in the total outer leaflet lipid was employed (Figure 6). With the increased amount of DSPE-PEG up to 23\%, the LCP-II formulation showed less RES uptake and preferred to accumulate in the tumor (Figure 6).

Quantitative analysis of the bio-distribution data shown in Figure 6 was analyzed by using the Image J software as described in section 2.6. The results showed that the ratio of tumor/ liver in animals treated with $23 \%$ PEG coated LCP-II was $1.58 \pm 0.14$, while the ones treated with $16 \%$ PEG coated LCP-II was $1.09 \pm 0.15$ ( $\mathrm{p}=0.03$ ). We conclude that higher levels of PEGylation of LCP-II favor tumor accumulation in the liver. The reason is probably because of the smaller particle size $(\sim 40 \mathrm{~nm})$, and hence higher curvature, of LCP-II as compared to 
LPD $(\sim 150 \mathrm{~nm})$. For equal densities of PEG, a high curvature surface would allow more polymer freedom of motion, and therefore less brush activity, than a low curvature surface.

\subsection{In vivo luciferase silencing effect}

To examine the silencing activity of siRNA delivered by LCP-IIin vivo, luciferase levels in the $\mathrm{H} 460$ xenograft tumor were detected after a single tail vein injection of NP containing luciferase siRNA (Figure 7).

LCP-II formulations prepared with either DOPC or DOTAP as the outer leaflet lipid were studied. All formulations contained DSPE-PEG-AA as the targeting ligand. With the siRNA dose of $0.12 \mathrm{mg} / \mathrm{kg}$, LCP-II prepared with DOPC as the outer leaflet lipid showed no silencing effect. The formulation containing DOTAP could down-regulate the luciferase activity to approximately $50 \%$ percent when the dose of siRNA was $0.6 \mathrm{mg} / \mathrm{kg}$. Sixty percent down-regulation could be reached at $1.2 \mathrm{mg} / \mathrm{kg}$ of siRNA dose. However, the luciferase activity did not change if control siRNA was delivered at the same dose. Thus, the estimated $\mathrm{ED}_{50}$ for LCP-II mediated delivery of siRNA was about $0.6 \mathrm{mg} / \mathrm{kg}$. Compared to LCP-I (estimated $\mathrm{ED}_{50}=1.2 \mathrm{mg} / \mathrm{kg}$ ) using the same xenograft tumor system and luciferase siRNA [15], the $\mathrm{ED}_{50}$ for siRNA delivery showed a modest improvement with LCP-II. Effective siRNA doses for gene silencing by using other delivery systems are generally larger than $1 \mathrm{mg} / \mathrm{kg}[12,27]$. Thus, both LCP NP formulations represent one of the best delivery vehicles for siRNA to the solid tumor.

There are two widely accepted major mechanisms for NP-mediated cargo release from the endosome. The first is the proton sponge effect in which cationic polymers containing $2^{\circ}$ and $3^{\circ}$ amines increase the osmotic pressure in the acidic endosome by a buffering effect[28], and cargo release is the result of the endosome bursting [29]. The second is the ion-pair formation between the positively charged groups of either cationic polymer or lipid and the negatively charged groups of the endosome membrane [30]. Clustered ion-pairs lead to relatively large areas of dehydration at the endosome membrane surface, resulting in destabilization of both the endosome membrane and the cationic vector. We have proposed a third mechanism in which osmotic pressure increase can be the result of $\mathrm{CaP}$ dissolution in the acidic endosome [15]. This mechanism obviously will require a sufficient number of LCP simultaneously internalized into the same endosome. If so, the release mechanism would not depend on the outer leaflet lipid. This was apparently the case for the in vitro condition in which a large number of LCP-II could be delivered to cells at the same time. Data in Figure 5 support this mechanism in that siRNA delivery by LCP-II containing either a neutral (DOPC) or a cationic lipid (DOTAP) showed equal silencing effect. However, data in Figure 7 clearly indicate that the LCP-II containing cationic lipid (DOTAP) delivered siRNA more efficiently than the one containing neutral lipid (DOPC).Thus, when an insufficient amount of NP is delivered to the same endosome at the same time, which is highly likely for the in vivo situation, cationic lipid is still important, probably for the formation of ion-pairs.

\section{Conclusion}

$\mathrm{CaP}$ core stabilized with DOPA was prepared by water/oil microemulsion and further coated with cationic or neutral lipid to form LCP-II. The vehicle has a hollow spherical structure with a size of about $40 \mathrm{~nm}$ and possesses an asymmetric lipid bilayer at the surface. With the targeting ligand anisamide, the new LCP-II showed a 40-fold improved silence activity compared to the previous LPD formulation. The new NP vehicle effectively delivers siRNA to solid tumor in a xenograft model. The therapeutic activity of the encapsulated siRNA will be tested in future experiments. 


\section{Supplementary Material}

Refer to Web version on PubMed Central for supplementary material.

\section{Acknowledgments}

This study was supported by NIH grants CA129835, CA149363 and CA151652.We thank Andrew Satterlee for editing the manuscript.

\section{List of abbreviations}

$\begin{array}{ll}\text { CAP } & \text { Calcium phosphate } \\ \text { DOPA } & \text { Dioleoylphosphatydic acid } \\ \text { DOPC } & \text { Dioleoylphosphatidylcholine } \\ \text { DOTAP } & \text { 1, 2-dioleoyl-3-trimethylammonium-propane chloride salt } \\ \text { DSPE-PEG } & \text { 1,2-distearoryl-sn-glycero-3-phosphoethanolamine-N- } \\ & \text { [methoxy(polyethyleneglycol-2000)] ammonium salt } \\ \text { DSPE-PEG-AA } & \text { DSPE-PEG- anisamide } \\ \text { LPD } & \text { Lipid/protamine/DNA } \\ \text { LCP } & \text { Lipid/Calcium/Phosphate } \\ \text { NP } & \text { Nanoparticle } \\ \text { MPS } & \text { Mononuclear phagocytic system }\end{array}$

\section{References}

1. Perkel JM. RNAi THERAPEUTICS: A TWO-YEAR UPDATE. Science. 2009; 326(5951):454456.

2. Whitehead KA, Langer R, Anderson DG. Knocking down barriers: advances in siRNA delivery. Nat Rev Drug Discov. 2009; 8(2):129-138. [PubMed: 19180106]

3. Li SD, Huang L. Nanoparticles evading the reticuloendothelial system: role of the supported bilayer. Biochim Biophys Acta. 2009; 1788(10):2259-2266. [PubMed: 19595666]

4. Davis ME. The first targeted delivery of siRNA in humans via a self-assembling, cyclodextrin polymer-based nanoparticle: from concept to clinic. Mol Pharm. 2009; 6(3):659-668. [PubMed: 19267452]

5. Semple SC, Akinc A, Chen J. Rational design of cationic lipids for siRNA delivery. Nat Biotechnol. 2010; 28(2):172-176. e. al. [PubMed: 20081866]

6. Love KT, Mahon KP, Levins CG. Lipid-like materials for low-dose, in vivo gene silencing. Proc Natl Acad Sci U S A. 2010; 107(5):1864-1869. e. al. [PubMed: 20080679]

7. Akinc A, Querbes W, De S. Targeted delivery of RNAi therapeutics with endogenous and exogenous ligand-based mechanisms. Mol Ther. 2010; 18(7):1357-1364. e. al. [PubMed: 20461061]

8. Akinc A, Goldberg M, Qin J. Development of lipidoid-siRNA formulations for systemic delivery to the liver. Mol Ther. 2009; 17(5):872-879. e. al. [PubMed: 19259063]

9. Chono S, Li SD, Conwell CC, Huang L. An efficient and low immunostimulatory nanoparticle formulation for systemic siRNA delivery to the tumor. J Control Release. 2008; 131(1):64-69. [PubMed: 18674578]

10. Li SD, Chen YC, Hackett MJ, Huang L. Tumor-targeted delivery of siRNA by self-assembled nanoparticles. Mol Ther. 2008; 16(1):163-169. [PubMed: 17923843]

11. Li SD, Chono S, Huang L. Efficient oncogene silencing and metastasis inhibition via systemic delivery of siRNA. Mol Ther. 2008; 16(5):942-946. [PubMed: 18388916] 
12. Davis ME, Zuckerman JE, Choi CH, Seligson D, Tolcher A, Alabi CA, Yen Y, Heidel JD, Ribas A. Evidence of RNAi in humans from systemically administered siRNA via targeted nanoparticles. Nature. 2010; 464(7291):1067-1070. [PubMed: 20305636]

13. Popielarski SR, Pun SH, Davis ME. A nanoparticle-based model delivery system to guide the rational design of gene delivery to the liver. 1. Synthesis and characterization. Bioconjug Chem. 2005; 16(5):1063-1070. [PubMed: 16173781]

14. Hu-Lieskovan S, Heidel JD, Bartlett DW, Davis ME, Triche TJ. Sequence-specific knockdown of EWS-FLI1 by targeted, nonviral delivery of small interfering RNA inhibits tumor growth in a murine model of metastatic Ewing's sarcoma. Cancer Res. 2005; 65(19):8984-8992. [PubMed: 16204072]

15. Li J, Chen YC, Tseng YC, Mozumdar S, Huang L. Biodegradable calcium phosphate nanoparticle with lipid coating for systemic siRNA delivery. J Control Release. 2010; 142(3):416-421. [PubMed: 19919845]

16. Alexis F, Pridgen E, Molnar LK, Farokhzad OC. Factors affecting the clearance and biodistribution of polymeric nanoparticles. Mol Pharm. 2008; 5(4):505-515. [PubMed: 18672949]

17. Uster PS, Allen TM, Daniel BE, Mendez CJ, Newman MS, Zhu GZ. Insertion of poly(ethylene glycol) derivatized phospholipid into pre-formed liposomes results in prolonged in vivo circulation time. FEBS Lett. 1996; 386(2-3):243-246. [PubMed: 8647291]

18. Cotmore JM, Nichols G Jr. Wuthier RE. Phospholipid-calcium phosphate complex: enhanced calcium migration in the presence of phosphate. Science. 1971; 172(990):1339-1341. [PubMed: 5580215]

19. Whittenton J, Harendra S, Pitchumani R, Mohanty K, Vipulanandan C, Thevananther S. Evaluation of asymmetric liposomal nanoparticles for encapsulation of polynucleotides. Langmuir. 2008; 24(16):8533-8540. [PubMed: 18597508]

20. Frederic Duconge TP. Carine Pestou oise Hinnen, Bertrand Kühnast, Frederic Dolle, Benoît Dubertret, Bertrand Tavitian, Fluorine-18-Labeled Phospholipid Quantum Dot Micelles for in Vivo Multimodal Imaging from Whole Body to Cellular Scales. Bioconjugate Chem. 2008; 19(9): 1921-1926.

21. Benoit Dubertret PS, Norris David J. Noireaux Vincent, Brivanlou Ali H. Libchaber Albert. In Vivo Imaging of Quantum Dots Encapsulated in Phospholipid Micelles. Science. 2002; 298(5599):1759-1762. [PubMed: 12459582]

22. Woodle MC, Lasic DD. Sterically stabilized liposomes. Biochim Biophys Acta. 1992; 1113(2): 171-199. [PubMed: 1510996]

23. Klibanov AL, Maruyama K, Torchilin VP, Huang L. Amphipathic polyethyleneglycols effectively prolong the circulation time of liposomes. FEBS Lett. 1990; 268(1):235-237. [PubMed: 2384160]

24. Banerjee R, Tyagi P, Li S, Huang L. Anisamide-targeted stealth liposomes: a potent carrier for targeting doxorubicin to human prostate cancer cells. Int J Cancer. 2004; 112(4):693-700. [PubMed: 15382053]

25. Garbuzenko O, Barenholz Y, Priev A. Effect of grafted PEG on liposome size and on compressibility and packing of lipid bilayer. Chem Phys Lipids. 2005; 135(2):117-129. [PubMed: 15921973]

26. Tan Y, Whitmore M, Li S, Frederik P, Huang L. LPD nanoparticles--novel nonviral vector for efficient gene delivery. Methods Mol Med. 2002; 69:73-81. [PubMed: 11987799]

27. Judge AD, Robbins M, Tavakoli I, Levi J, Hu L, Fronda A, Ambegia E, McClintock K, MacLachlan I. Confirming the RNAi-mediated mechanism of action of siRNA-based cancer therapeutics in mice. J Clin Invest. 2009; 119(3):661-673. [PubMed: 19229107]

28. Boussif O, Lezoualc'h F, Zanta MA, Mergny MD, Scherman D, Demeneix B, Behr JP. A versatile vector for gene and oligonucleotide transfer into cells in culture and in vivo: polyethylenimine. Proc Natl Acad Sci U S A. 1995; 92(16):7297-7301. [PubMed: 7638184]

29. Xu Y, Szoka FC Jr. Mechanism of DNA release from cationic liposome/DNA complexes used in cell transfection. Biochemistry. 1996; 35(18):5616-5623. [PubMed: 8639519]

30. Hafez IM, Maurer N, Cullis PR. On the mechanism whereby cationic lipids promote intracellular delivery of polynucleic acids. Gene Ther. 2001; 8(15):1188-1196. [PubMed: 11509950] 

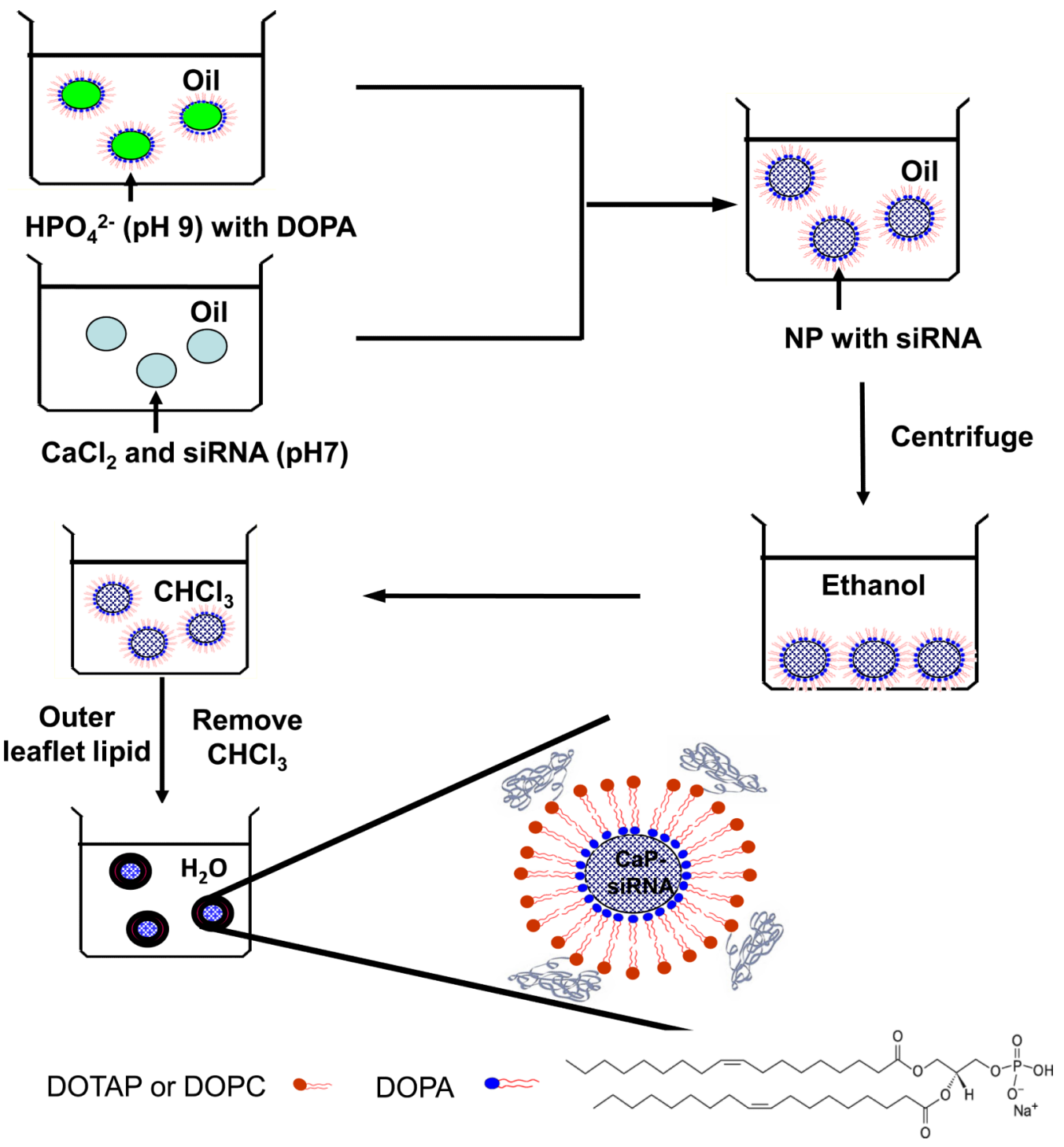

Figure 1.

The outline for the preparation of LCP-II NP and the structure of DOPA. 

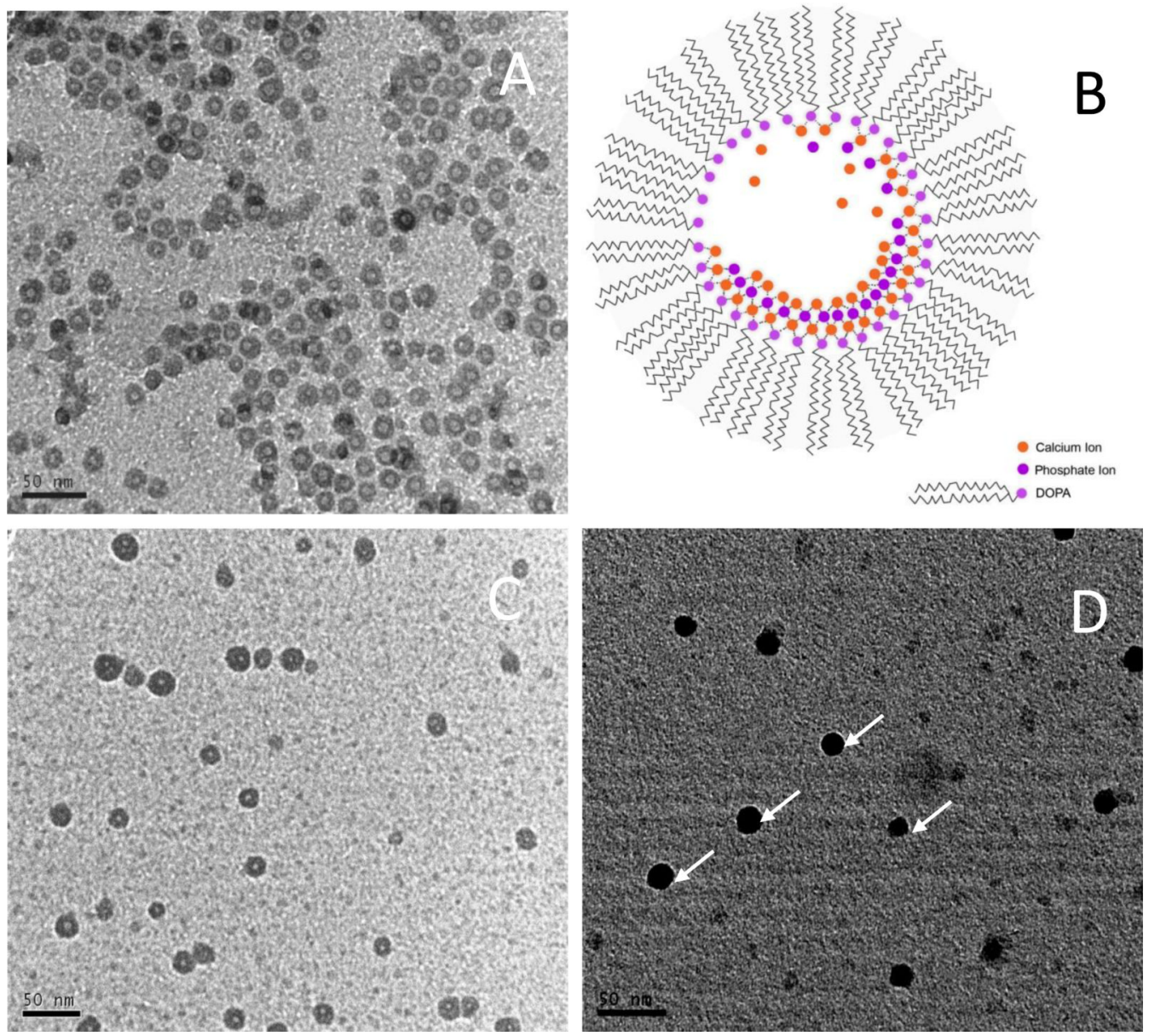

Figure 2.

(A), TEM image of $\mathrm{CaP}$ cores coated with DOPA. (B), Hypothesis of the $\mathrm{CaP}$ core growth. (C) and (D),TEM images of LCP-II NPs coated with DOTAP and DSPE-PEG without(C) and with (D) negative staining. Arrows in (D) show lipid bilayer surrounding the $\mathrm{CaP}$ core. 


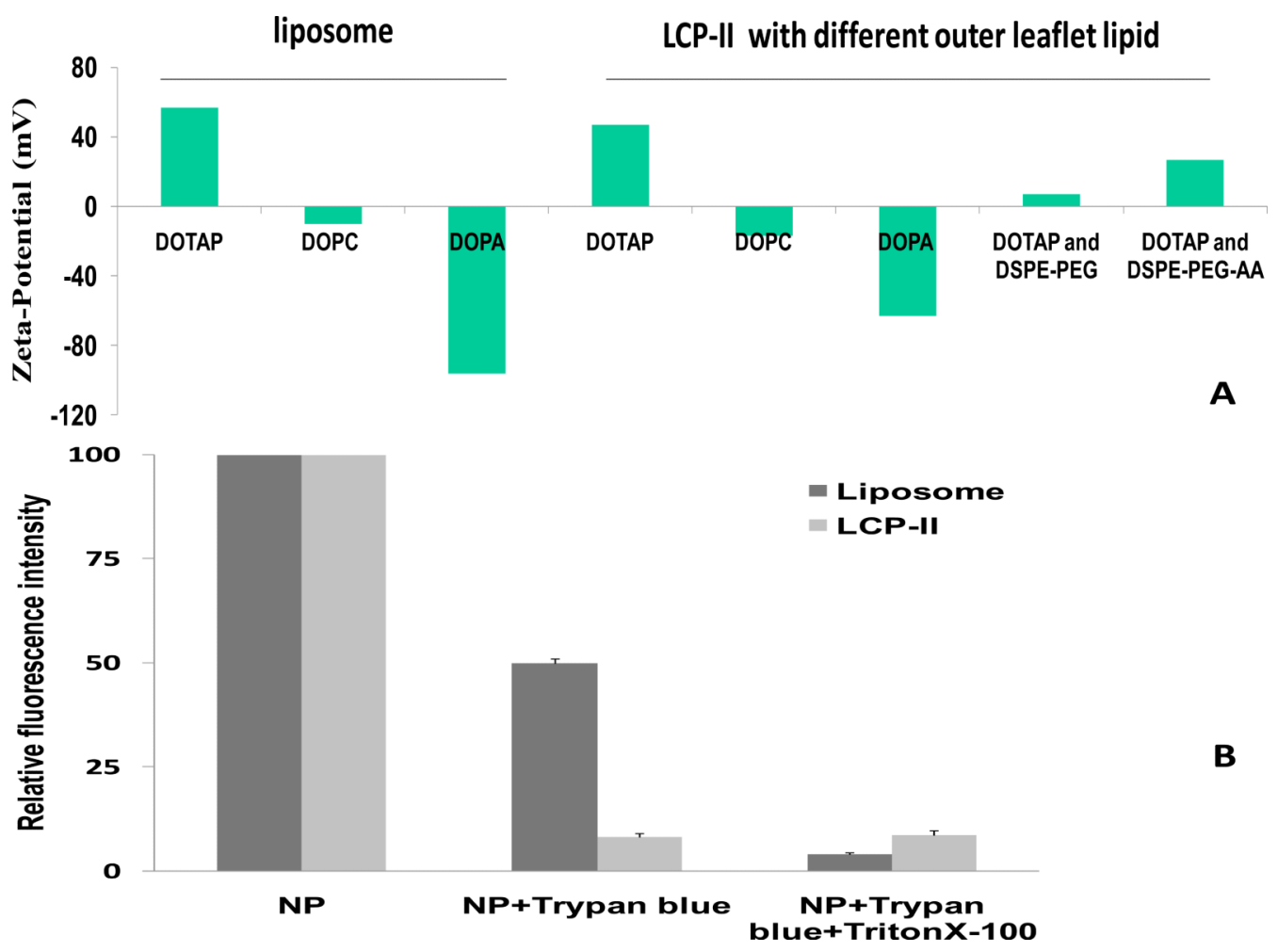

Figure 3.

(A) Zeta-potential of different liposome and LCP-II formulations. All lipid compositions contained equal amount of cholesterol. (B) Fluorescence quenching of Rhod-PE by trypan blue. $\mathrm{N}=3$. 

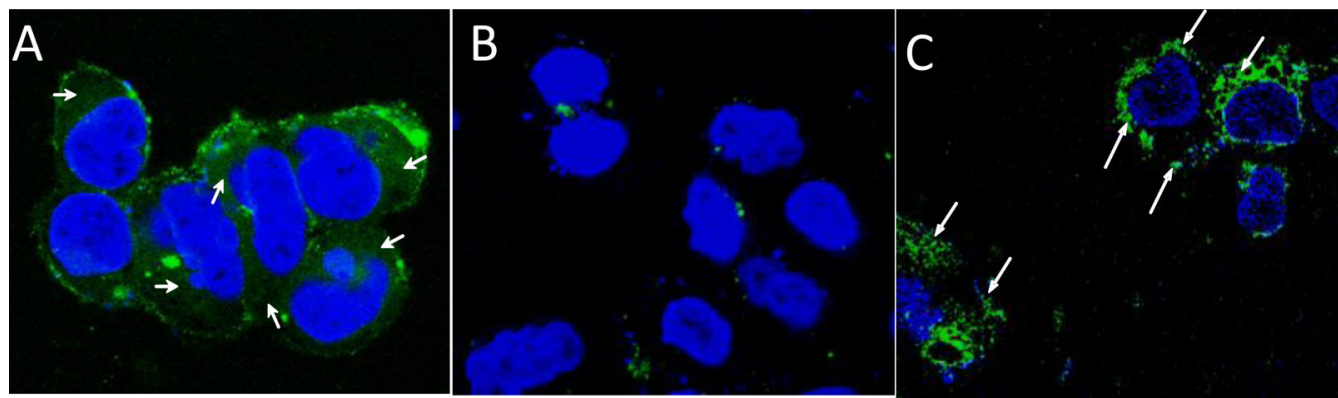

Figure 4.

Subcellular distribution of FAM-labeled dsDNA (model for siRNA) in H460 cells. Cells were incubated with $50 \mathrm{nM}$ dsDNA formulated in LCP-II coated with DOTAP targeted with AA (A), untargeted LCP-II (B), or AA-targeted LPD (C) for $3 \mathrm{~h}$ and imaged with confocal microscopy. Short arrows in (A) indicate spread, even distribution of fluorescently labeled dsDNA. Long arrows in $(C)$ indicate punctuate distribution of dsDNA. 


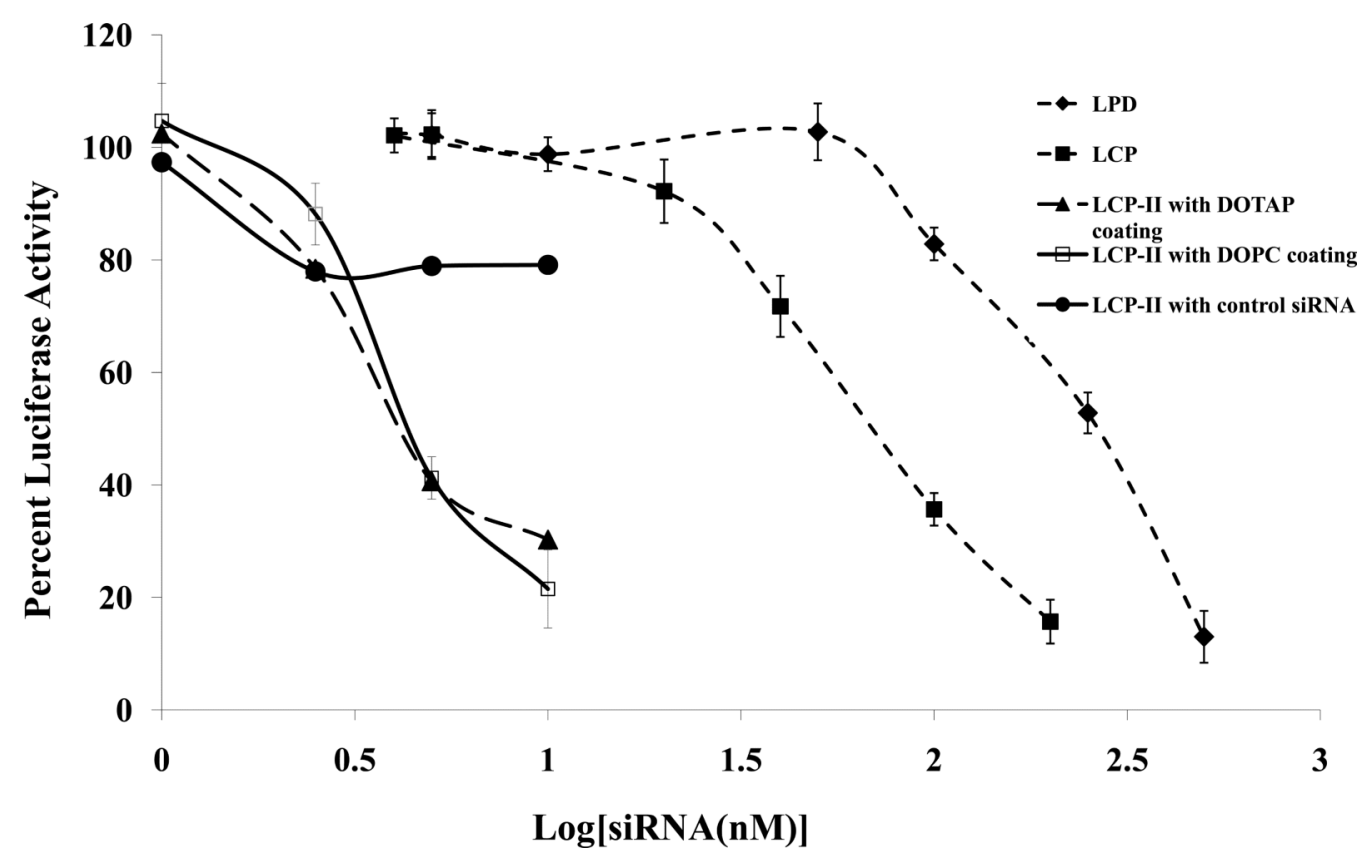

Figure 5 .

In vitro silencing effect of anti-luciferase siRNA formulated in LPD, LCP and LCP-II with DOTAP (dotted line) and DOPC (solid line) as the outer leaflet lipid. Data indicate that LCP-II was about 50-fold more active in delivering siRNA than LPD. 


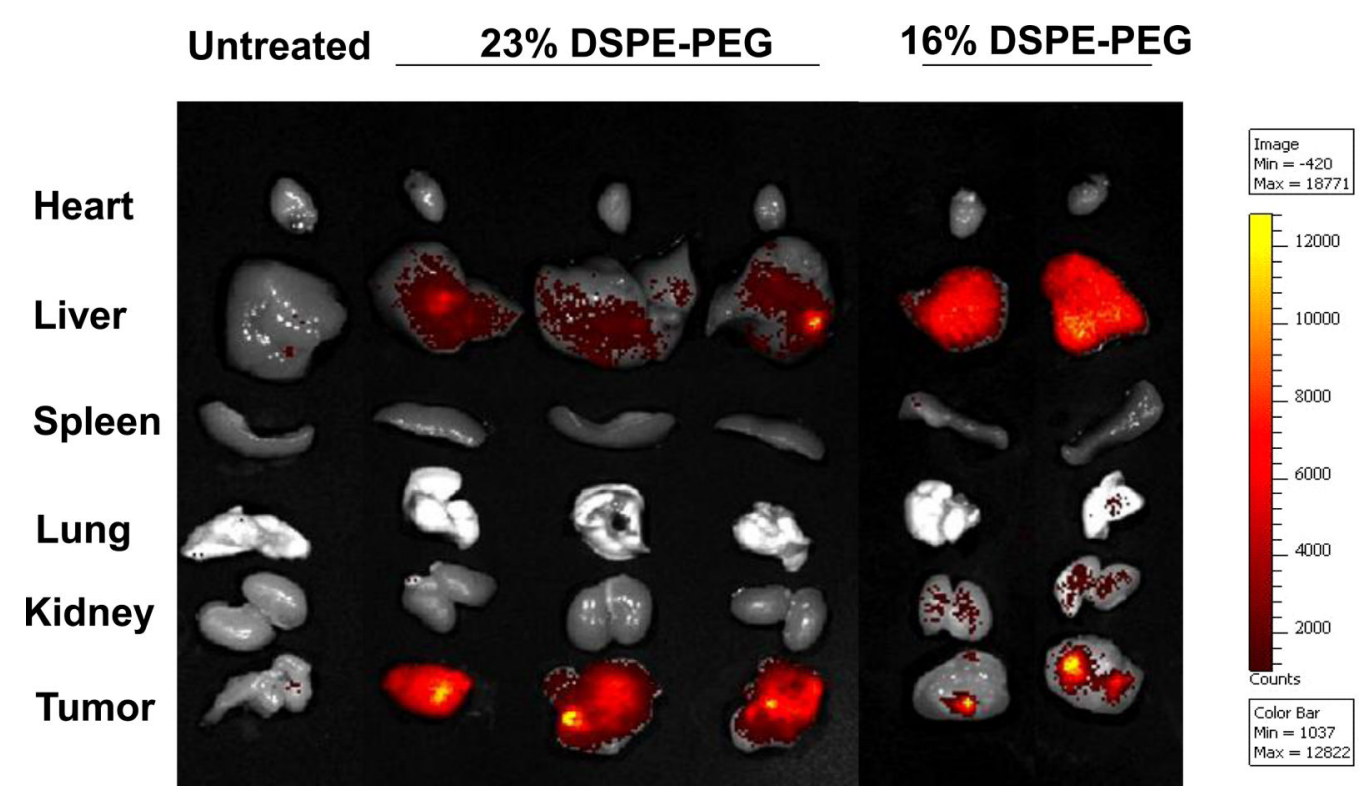

Figure 6.

Biodistribution of fluorescence-labeled siRNA delivered by LCP-II coated with DOTAP and modified with different amounts of DSPE-PEG. LCP-II was coated with either 16 or 23 mol $\%$ of DSPE-PEG and i.v. injected into nude mice bearing human H460 tumor. 


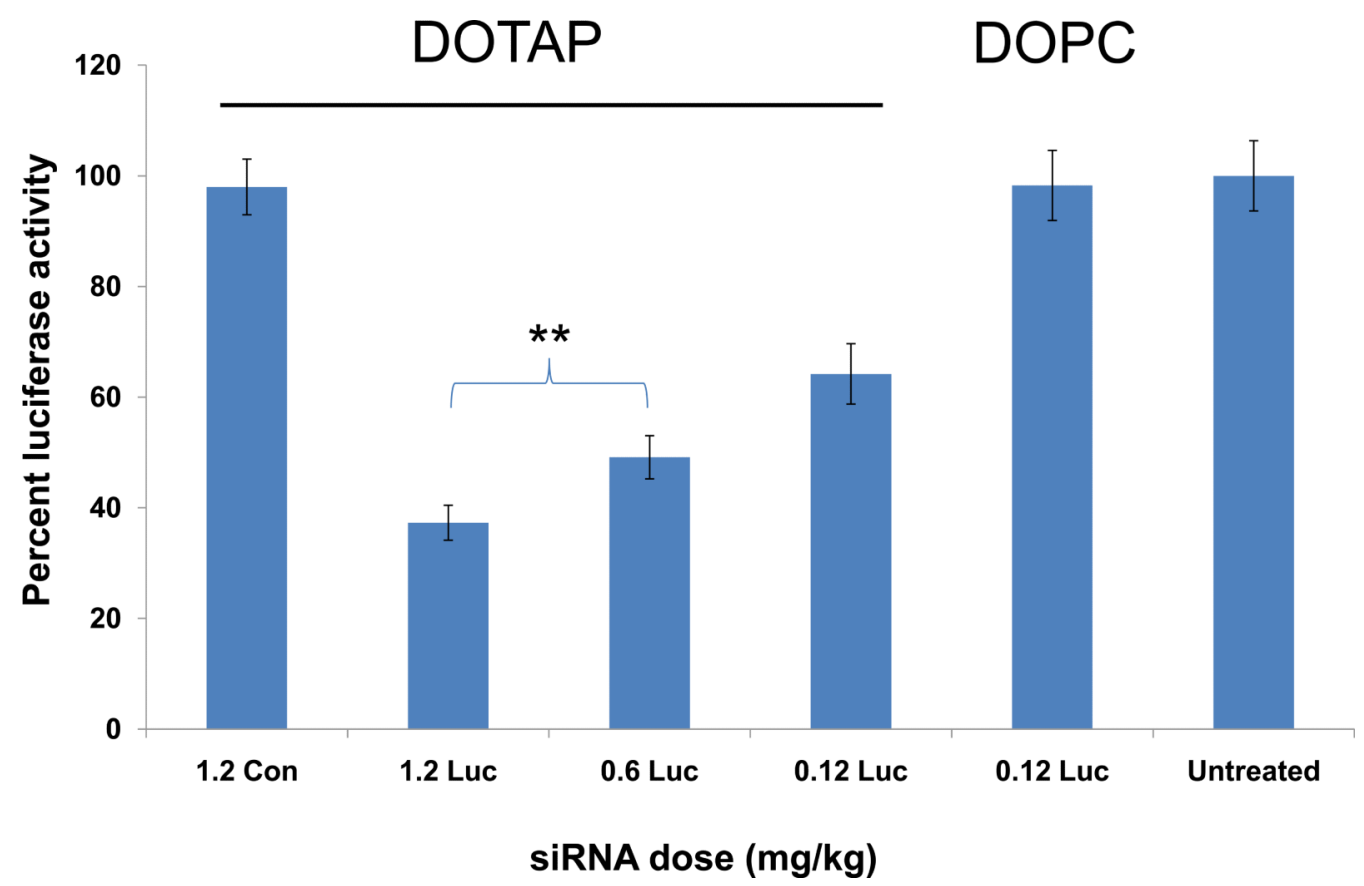

Figure 7.

In vivo silencing effect of luciferase siRNA delivered with LCP-II. Mice bearing human H460 tumor stably expressing luciferase were i.v. injected with LCP-II prepared with either DOTAP or DOPC, indicated at the top of the figure, as the outer leaflet lipid. Luc: luciferase siRNA. Con: control siRNA. Numbers in X-axis indicate the injected dose of siRNA in $\mathrm{mg}$ / kg. $* *$ indicates $\mathrm{p}<0.05$. 